\title{
HOCHSCHILD COHOMOLOGY RING OF THE GENERALIZED QUATERNION ALGEBRAS
}

\author{
By \\ Takao HAYAMI ${ }^{\dagger}$
}

\begin{abstract}
We will give an efficient bimodule projective resolution of the generalized quaternion $\mathbf{Z}$ algebra $\Gamma$. As a main result, we will determine the ring structure of the Hochschild cohomology $\mathrm{HH}^{*}(\Gamma)$ by calculating the Yoneda products using this resolution.
\end{abstract}

\section{Introduction}

Let $R$ be a commutative ring and $\Lambda$ an $R$-algebra which is a finitely generated projective $R$-module. If $M$ is a $\Lambda$-bimodule (i.e., a $\Lambda^{\mathrm{e}}=\Lambda \otimes_{R} \Lambda^{\mathrm{op}}$ module), then the $n$th Hochschild cohomology of $\Lambda$ with coefficients in $M$ is defined by $H^{n}(\Lambda, M):=\operatorname{Ext}_{\Lambda^{\mathrm{e}}}^{n}(\Lambda, M)$. If $M=\Lambda$, we set $H H^{n}(\Lambda)=H^{n}(\Lambda, \Lambda)$. The Yoneda product gives $H H^{*}(\Lambda):=\bigoplus_{n \geq 0} H H^{n}(\Lambda)$ a graded ring structure with $1 \in Z \Lambda \simeq H H^{0}(\Lambda)$ where $Z \Lambda$ denotes the center of $\Lambda$. $H H^{*}(\Lambda)$ is called the Hochschild cohomology ring of $\Lambda$ (see [4], [1], [5]). The Hochschild cohomology ring $H H^{*}(\Lambda)$ is a graded-commutative algebra, that is, $\alpha \beta=(-1)^{p q} \beta \alpha$ holds for $\alpha \in H H^{p}(\Lambda)$ and $\beta \in H H^{q}(\Lambda)$. The Hochschild cohomology is an important invariant of algebras, however the Hochschild cohomology ring is difficult to compute in general.

Suppose that $a$ and $b$ are any nonzero rational integers. We consider the generalized quaternion $\mathbf{Z}$ algebra $\Gamma:=\mathbf{Z} \oplus \mathbf{Z} i \oplus \mathbf{Z} j \oplus \mathbf{Z} i j$ with the relations $i^{2}=a, j^{2}=b, i j=-j i$. In the case $a=-1$ and $b=-1$, the ring structure of the Hochschild cohomology of the ordinary quaternion algebra $\Gamma$ is already known by Sanada [6, Section 3.4].

\footnotetext{
${ }^{\dagger}$ This research was partially supported by the scientific research grants from Hokkai-Gakuen University. 2000 Mathematics Subject Classification: 16E40.

Key words and phrases: Hochschild cohomology ring, bimodule projective resolution, generalized quaternion algebra, Yoneda product.

Received March 21, 2012.

Revised October 16, 2012.
} 
THEOREM (Sanada [6]). Let $\Gamma$ be the ordinary quaternion algebra over $\mathbf{Z}$. Then the Hochschild cohomology ring of $\Gamma$ is as follows:

$$
H H^{*}(\Gamma)=\mathbf{Z}[\lambda, \mu, v] /\left(2 \lambda, 2 \mu, 2 v, \lambda^{2}+\mu^{2}+v^{2}\right),
$$

where $\operatorname{deg} \lambda=\operatorname{deg} \mu=\operatorname{deg} v=1$.

In [2], the author reproves and generalizes this result. Thus it is natural question to investigate the ring structure of the Hochschild cohomology of the generalized quaternion algebra. In this article, we will give an efficient bimodule projective resolution of the generalized quaternion algebra $\Gamma$. Moreover by using this resolution, we determine the ring structure of the Hochschild cohomology ring of the generalized quaternion algebra $\Gamma$. This is a method similar to [2] or [3].

In Section 1, we state an efficient bimodule projective resolution of $\Gamma$ (Theorem 1.1). In Section 2, we use the resolution to describe the module structure of $H H^{*}(\Gamma)$, giving explicit generators (Theorem 2.1). In Section 3, we compute the Yoneda products of the generators. Then, as a main result of this article, we give a complete description of the Hochschild cohomology ring $H H^{*}(\Gamma)$ (Theorem 3.8). The result is more complicated than the known result for the ordinary quaternion algebra.

\section{Bimodule Projective Resolution for the Generalized Quaternion Algebras}

Suppose that $a$ and $b$ are any nonzero rational integers. Let

$$
\Gamma:=\mathbf{Z} \oplus \mathbf{Z} i \oplus \mathbf{Z} j \oplus \mathbf{Z} i j
$$

be the generalized quaternion algebra over $\mathbf{Z}$ with the relations $i^{2}=a, j^{2}=b$, $i j=-j i$.

In the following, we give an efficient bimodule projective resolution of $\Gamma$. For each integer $q \geq 0$, let $Y_{q}$ be the direct sum of $q+1$ copies of $\Gamma \otimes \Gamma$. We define elements of $Y_{q}$ by

$$
c_{q}^{s}= \begin{cases}(0, \ldots, 0,1 \stackrel{s}{\otimes} 1,0, \ldots, 0) & (\text { if } 1 \leq s \leq q+1), \\ 0 & \text { (otherwise). }\end{cases}
$$

Then we have that $Y_{q}=\bigoplus_{k=1}^{q+1} \Gamma c_{q}^{k} \Gamma$.

THEOREM 1.1. There exists the following bimodule projective resolution of $\Gamma$ :

$$
(Y, \delta): \cdots \rightarrow Y_{3} \stackrel{\delta_{3}}{\rightarrow} Y_{2} \stackrel{\delta_{2}}{\rightarrow} Y_{1} \stackrel{\delta_{1}}{\rightarrow} Y_{0} \stackrel{\delta_{0}}{\rightarrow} \Gamma \rightarrow 0
$$


where $\delta_{0}: Y_{0} \rightarrow \Gamma$ is the multiplication map, and for integer $q>0, \delta_{q}: Y_{q} \rightarrow Y_{q-1}$ is a $\Gamma^{\mathrm{e}}$-homomorphism given by

$$
\delta_{q}\left(c_{q}^{s}\right)= \begin{cases}i c_{q-1}^{s}-c_{q-1}^{s} i+j c_{q-1}^{s-1}-c_{q-1}^{s-1} j & \text { for } q \text { odd }, \\ i c_{q-1}^{s}+c_{q-1}^{s} i+j c_{q-1}^{s-1}+c_{q-1}^{s-1} j & \text { for } q \text { even } .\end{cases}
$$

Proof. By direct computations, we have that $\delta_{q} \cdot \delta_{q+1}=0$ for $q \geq 0$. For example, if $q(\geq 1)$ is odd, then we have

$$
\begin{aligned}
\delta_{q} \cdot \delta_{q+1}\left(c_{q+1}^{s}\right)= & \delta_{q}\left(i c_{q}^{s}+c_{q}^{s} i+j c_{q}^{s-1}+c_{q}^{s-1} j\right) \\
= & a c_{q-1}^{s}-i c_{q-1}^{s} i+i j c_{q-1}^{s-1}-i c_{q-1}^{s-1} j \\
& +i c_{q-1}^{s} i-c_{q-1}^{s} a+j c_{q-1}^{s-1} i-c_{q-1}^{s-1} j i \\
& +j i c_{q-1}^{s-1}-j c_{q-1}^{s-1} i+b c_{q-1}^{s-2}-j c_{q-1}^{s-2} j \\
& +i c_{q-1}^{s-1} j-c_{q-1}^{s-1} i j+j c_{q-1}^{s-2} j-c_{q-1}^{s-2} b \\
= & 0 .
\end{aligned}
$$

Thus we have $\operatorname{Im} \delta_{q+1} \subset \operatorname{Ker} \delta_{q}$ for $q \geq 0$.

Next we prove the reverse inclusion. We give a contracting homotopy. We define right $\Gamma$-homomorphisms $T_{-1}: \Gamma \rightarrow Y_{0}$ and $T_{q}: Y_{q} \rightarrow Y_{q+1}(q \geq 0)$ as follows:

$$
\begin{aligned}
T_{-1}(\gamma) & =c_{0}^{1} \gamma \quad(\forall \gamma \in \Gamma), \\
T_{q}\left(i^{m} j^{n} c_{q}^{s}\right) & = \begin{cases}m c_{q+1}^{1} & (q \geq 0, s=1, m=0,1, n=0), \\
(-1)^{q} m c_{q+1}^{1} j+i^{m} c_{q+1}^{2} & (q \geq 0, s=1, m=0,1, n=1), \\
0 & (q \geq 0, s \geq 2, m=0,1, n=0), \\
i^{m} c_{q+1}^{s+1} & (q \geq 0, s \geq 2, m=0,1, n=1) .\end{cases}
\end{aligned}
$$

We may see that $T_{q}: Y_{q} \rightarrow Y_{q+1}(q \geq-1)$ is a contracting homotopy. Thus we must check that the equation

$$
\left(\delta_{q+1} T_{q}+T_{q-1} \delta_{q}\right)\left(i^{m} j^{n} c_{q}^{s}\right)=i^{m} j^{n} c_{q}^{s}
$$

holds for $q \geq 0 ; 1 \leq s \leq q+1 ; m=0,1 ; n=0,1$.

If $q=0$, we have

$$
T_{-1} \delta_{0}\left(i^{m} j^{n} c_{0}^{1}\right)=T_{-1}\left(i^{m} j^{n}\right)=c_{0}^{1} i^{m} j^{n}
$$


where $m=0,1$ and $n=0,1$. On the other hand, if $n=0$, we have

$$
\delta_{1} T_{0}\left(i^{m} c_{0}^{1}\right)=\delta_{1}\left(m c_{1}^{1}\right)=m\left(i c_{0}^{1}-c_{0}^{1} i\right),
$$

where $m=0,1$. If $n=1$, we have

$$
\begin{aligned}
\delta_{1} T_{0}\left(i^{m} j c_{0}^{1}\right) & =\delta_{1}\left(m c_{1}^{1} j+i^{m} c_{1}^{2}\right) \\
& =m\left(i c_{0}^{1}-c_{0}^{1} i\right) j+i^{m}\left(j c_{0}^{1}-c_{0}^{1} j\right) \\
& =i^{m} j c_{0}^{1}-c_{0}^{1} i^{m} j,
\end{aligned}
$$

where $m=0,1$. Thus we have $\delta_{1} T_{0}+T_{-1} \delta_{0}=\mathrm{id}_{Y_{0}}$.

If $q(\geq 1)$ is odd, then for $s=1$ and $n=0$, we have

$$
\begin{aligned}
& \delta_{q+1} T_{q}\left(i^{m} c_{q}^{1}\right)=\delta_{q+1}\left(m c_{q+1}^{1}\right)=m\left(i c_{q}^{1}+c_{q}^{1} i\right) \quad(m=0,1), \\
& T_{q-1} \delta_{q}\left(i^{m} c_{q}^{1}\right)=T_{q-1}\left(i^{m+1} c_{q-1}^{1}-i^{m} c_{q-1}^{1} i\right)= \begin{cases}c_{q}^{1} & (m=0), \\
-c_{q}^{1} i & (m=1) .\end{cases}
\end{aligned}
$$

If $q(\geq 1)$ is odd, then for $s=1$ and $n=1$, we have

$$
\begin{aligned}
\delta_{q+1} T_{q}\left(i^{m} j c_{q}^{1}\right) & =\delta_{q+1}\left(-m c_{q+1}^{1} j+i^{m} c_{q+1}^{2}\right) \\
& = \begin{cases}j c_{q}^{1}+c_{q}^{1} j+i c_{q}^{2}+c_{q}^{2} i & (m=0), \\
i j c_{q}^{1}-c_{q}^{1} i j+a c_{q}^{2}+i c_{q}^{2} i & (m=1),\end{cases} \\
T_{q-1} \delta_{q}\left(i^{m} j c_{q}^{1}\right) & = \begin{cases}T_{q-1}\left(-i j c_{q-1}^{1}-j c_{q-1}^{1} i\right) & (m=0), \\
T_{q-1}\left(-a j c_{q-1}^{1}-i j c_{q-1}^{1} i\right) & (m=1)\end{cases} \\
& = \begin{cases}-c_{q}^{1} j-i c_{q}^{2}-c_{q}^{2} i & (m=0), \\
c_{q}^{1} i j-a c_{q}^{2}-i c_{q}^{2} i & (m=1) .\end{cases}
\end{aligned}
$$

If $q(\geq 1)$ is odd, then for $s \geq 2, m=0,1$, and $n=0$, we have

$$
\begin{aligned}
\delta_{q+1} T_{q}\left(i^{m} c_{q}^{s}\right) & =0 \\
T_{q-1} \delta_{q}\left(i^{m} c_{q}^{s}\right) & =T_{q-1}\left(i^{m+1} c_{q-1}^{s}-i^{m} c_{q-1}^{s} i+i^{m} j c_{q-1}^{s-1}-i^{m} c_{q-1}^{s-1} j\right) \\
& =i^{m} c_{q}^{s} .
\end{aligned}
$$

If $q(\geq 1)$ is odd, then for $s \geq 2$ and $n=1$, we have

$$
\begin{aligned}
\delta_{q+1} T_{q}\left(i^{m} j c_{q}^{s}\right) & =\delta_{q+1}\left(i^{m} c_{q+1}^{s+1}\right) \\
& =i^{m+1} c_{q}^{s+1}+i^{m} c_{q}^{s+1} i+i^{m} j c_{q}^{s}+i^{m} c_{q}^{s} j \quad(m=0,1),
\end{aligned}
$$




$$
\begin{aligned}
T_{q-1} \delta_{q}\left(i^{m} j c_{q}^{s}\right) & = \begin{cases}T_{q-1}\left(-i j c_{q-1}^{s}-j c_{q-1}^{s} i+b c_{q-1}^{s-1}-j c_{q-1}^{s-1} j\right) & (m=0), \\
T_{q-1}\left(-a j c_{q-1}^{s}-i j c_{q-1}^{s} i+b i c_{q-1}^{s-1}-i j c_{q-1}^{s-1} j\right) & (m=1)\end{cases} \\
& = \begin{cases}-i c_{q}^{s+1}-c_{q}^{s+1} i-c_{q}^{s} j & (m=0), \\
-a c_{q}^{s+1}-i c_{q}^{s+1} i-i c_{q}^{s} j & (m=1) .\end{cases}
\end{aligned}
$$

Thus we have $\delta_{q+1} T_{q}+T_{q-1} \delta_{q}=\mathrm{id}_{Y_{q}}$ for $q(\geq 1)$ odd. In the case $q(\geq 2)$ even, the computations are similar. Hence $\operatorname{Im} \delta_{q+1} \supset \operatorname{Ker} \delta_{q}$ is proved for $q \geq 0$.

REMARK 1.2. If $a=-1$ and $b=-1$, an efficient bimodule projective resolution of the ordinary quaternion algebra $\Gamma$ is given in [2]. Even in that special situation, the differential of the resolution in Theorem 1.1 is different from that of [2, Theorem 1.1].

\section{Module Structure}

We keep the notations in Section 1. In this section, we calculate the Hochschild cohomology group $H H^{*}(\Gamma)$.

Let $M^{q}$ denote the direct sum of $q$ copies of a module $M$ for any integer $q>0$. As elements of $\Gamma^{q+1}$, we set

$$
l_{q}^{s}= \begin{cases}(0, \ldots, 0, \stackrel{s}{1}, 0, \ldots, 0) & (\text { if } 1 \leq s \leq q+1), \\ 0 & \text { (otherwise) }\end{cases}
$$

Then we have $\Gamma^{q+1}=\bigoplus_{k=1}^{q+1} \Gamma l_{q}^{k}$.

Applying the functor $\operatorname{Hom}_{\Gamma^{\mathrm{e}}}(-, \Gamma)$ to the resolution $(Y, \delta)$, we have the following complex, where we identify $\operatorname{Hom}_{\Gamma^{\mathrm{e}}}\left(Y_{q}, \Gamma\right)$ with $\Gamma^{q+1}$ using an isomorphism $\operatorname{Hom}_{\Gamma^{\mathrm{e}}}\left(Y_{q}, \Gamma\right) \rightarrow \Gamma^{q+1} ; f \mapsto \sum_{k=1}^{q+1} f\left(c_{q}^{k}\right) l_{q}^{k}$ :

$$
\begin{gathered}
\left(\operatorname{Hom}_{\Gamma^{\mathrm{e}}}(Y, \Gamma), \delta^{\#}\right): 0 \longrightarrow \Gamma \stackrel{\delta_{1}^{\#}}{\longrightarrow} \Gamma^{2} \stackrel{\delta_{2}^{\#}}{\longrightarrow} \Gamma^{3} \stackrel{\delta_{3}^{\#}}{\longrightarrow} \Gamma^{4} \stackrel{\delta_{4}^{\#}}{\longrightarrow} \Gamma^{5} \longrightarrow \cdots, \\
\delta_{q+1}^{\#}\left(\gamma l_{q}^{s}\right)= \begin{cases}(i \gamma-\gamma i) l_{q+1}^{s}+(j \gamma-\gamma j) l_{q+1}^{s+1} & \text { for } q \text { odd }, \\
(i \gamma+\gamma i) l_{q+1}^{s}+(j \gamma+\gamma j) l_{q+1}^{s+1} & \text { for } q \text { even } .\end{cases}
\end{gathered}
$$

In the above, note that

$$
\gamma l_{q}^{s}= \begin{cases}(0, \ldots, 0, \stackrel{s}{\gamma}, 0, \ldots, 0) & (\text { if } 1 \leq s \leq q+1) \\ 0 & (\text { otherwise })\end{cases}
$$

for $\gamma \in \Gamma$, and so on. 
In the following we set

$$
d=\operatorname{gcd}(a, b), \quad a^{\prime}=\frac{a}{d}, \quad b^{\prime}=\frac{b}{d} .
$$

Then we have the following theorem.

THEOREM 2.1. The Hochschild cohomology group of $\Gamma$ is as follows:

$$
H H^{n}(\Gamma)= \begin{cases}\mathbf{Z} & (n=0), \\ (\mathbf{Z} / 2 \mathbf{Z})^{n+1} \oplus(\mathbf{Z} / 2 d \mathbf{Z})^{n} & (n \text { odd }), \\ \mathbf{Z} / 2 a \mathbf{Z} \oplus(\mathbf{Z} / 2 d \mathbf{Z})^{n-1} \oplus \mathbf{Z} / 2 b \mathbf{Z} \oplus(\mathbf{Z} / 2 \mathbf{Z})^{n} & (n(\neq 0) \text { even }) .\end{cases}
$$

Furthermore, module generators of $H H^{n}(\Gamma)$ are given as follows:

(i) If $n(\geq 1)$ is odd, the $(\mathbf{Z} / 2 \mathbf{Z})^{n+1}$ summands are generated by $i j l_{n}^{k}$ $(k=1,2, \ldots, n+1)$ and the $(\mathbf{Z} / 2 d \mathbf{Z})^{n}$ summands are generated by $a^{\prime} j l_{n}^{k}-b^{\prime} i i_{n}^{k+1}(k=1,2, \ldots, n)$.

(ii) If $n(\geq 2)$ is even, the $\mathbf{Z} / 2 a \mathbf{Z}$ summand is generated by $\imath_{n}^{1}$, the $(\mathbf{Z} / 2 d \mathbf{Z})^{n-1}$ summands are generated by $l_{n}^{k}(k=2,3, \ldots, n)$, the $\mathbf{Z} / 2 b \mathbf{Z}$ summand is generated by $\imath_{n}^{n+1}$, and the $(\mathbf{Z} / 2 \mathbf{Z})^{n}$ summands are generated by $i l_{n}^{k}+j l_{n}^{k+1}(k=1,2, \ldots, n)$.

Proof. For any element $\gamma=x+y i+z j+w i j(x, y, z, w \in \mathbf{Z})$ in $\Gamma$, we have

$$
\begin{array}{ll}
i \gamma=x i+a y+z i j+a w j, & \gamma i=x i+a y-z i j-a w j, \\
j \gamma=x j-y i j+b z-b w i, & \gamma j=x j+y i j+b z+b w i .
\end{array}
$$

We prove the case $n(>0)$ even only. Let $\left(\gamma_{1}, \gamma_{2}, \ldots, \gamma_{n+1}\right)$ be any element in $\Gamma^{n+1}$ where we set $\gamma_{k}=x_{k}+y_{k} i+z_{k} j+w_{k} i j\left(x_{k}, y_{k}, z_{k}, w_{k} \in \mathbf{Z}\right)$. Since

$$
\begin{aligned}
& \left(\gamma_{1}, \gamma_{2}, \ldots, \gamma_{n+1}\right) \in \operatorname{Ker} \delta_{n+1}^{\#} \\
& \quad \Leftrightarrow\left\{\begin{array}{l}
2 z_{1} i j+2 a w_{1} j=0, \\
-2 y_{k} i j-2 b w_{k} i+2 z_{k+1} i j+2 a w_{k+1} j=0 \quad(k=1,2, \ldots, n), \\
-2 y_{n+1} i j-2 b w_{n+1} i=0
\end{array}\right. \\
& \quad \Leftrightarrow \begin{cases}z_{1}=y_{n+1}=0, & (k=1,2, \ldots, n+1), \\
w_{k}=0 & (k=1,2, \ldots, n) \\
y_{k}=z_{k+1}\end{cases} \\
& \Leftrightarrow\left(\gamma_{1}, \gamma_{2}, \ldots, \gamma_{n+1}\right)=\sum_{k=1}^{n+1} x_{k} l_{n}^{k}+\sum_{k=1}^{n} y_{k}\left(i l_{n}^{k}+j l_{n}^{k+1}\right),
\end{aligned}
$$


we have

$$
\operatorname{Ker} \delta_{n+1}^{\#}=\bigoplus_{k=1}^{n+1} \mathbf{Z} \imath_{n}^{k} \oplus \bigoplus_{k=1}^{n} \mathbf{Z}\left(i l_{n}^{k}+j l_{n}^{k+1}\right)
$$

Next we show

$$
\operatorname{Im} \delta_{n}^{\#}=2 a \mathbf{Z} \imath_{n}^{1} \oplus \bigoplus_{k=2}^{n} 2 d \mathbf{Z} \imath_{n}^{k} \oplus 2 b \mathbf{Z} \imath_{n}^{n+1} \oplus \bigoplus_{k=1}^{n} 2 \mathbf{Z}\left(i l_{n}^{k}+j l_{n}^{k+1}\right) .
$$

Let $\left(\gamma_{1}, \gamma_{2}, \ldots, \gamma_{n}\right)$ be any element in $\Gamma^{n}$ where we set $\gamma_{k}=x_{k}+y_{k} i+z_{k} j+w_{k} i j$ $\left(x_{k}, y_{k}, z_{k}, w_{k} \in \mathbf{Z}\right)$. Then we have

$$
\begin{aligned}
& \delta_{n}^{\#}\left(\gamma_{1}, \gamma_{2}, \ldots, \gamma_{n}\right) \\
& \quad=2 a y_{1} l_{n}^{1}+2 \sum_{k=2}^{n}\left(a y_{k}+b z_{k-1}\right) l_{n}^{k}+2 b z_{n} l_{n}^{n+1}+2 \sum_{k=1}^{n} x_{k}\left(i l_{n}^{k}+j l_{n}^{k+1}\right) \\
& \quad=2 a y_{1} l_{n}^{1}+2 d \sum_{k=2}^{n}\left(a^{\prime} y_{k}+b^{\prime} z_{k-1}\right) l_{n}^{k}+2 b z_{n} l_{n}^{n+1}+2 \sum_{k=1}^{n} x_{k}\left(i l_{n}^{k}+j l_{n}^{k+1}\right) .
\end{aligned}
$$

Note that $a^{\prime} y_{k}+b^{\prime} z_{k-1}$ is to be any element of $\mathbf{Z}$ by choosing $y_{k}$ and $z_{k-1}$ properly. Hence we have

$$
H H^{n}(\Gamma)=(\mathbf{Z} / 2 a \mathbf{Z}) \iota_{n}^{1} \oplus \bigoplus_{k=2}^{n}(\mathbf{Z} / 2 d \mathbf{Z}) \iota_{n}^{k} \oplus(\mathbf{Z} / 2 b \mathbf{Z}) \iota_{n}^{n+1} \oplus \bigoplus_{k=1}^{n}(\mathbf{Z} / 2 \mathbf{Z})\left(i u_{n}^{k}+j l_{n}^{k+1}\right)
$$

The other cases are similar.

REMARK 2.2. In particular if $a= \pm 1, b= \pm 1$, then we have that

$$
H H^{n}(\Gamma)= \begin{cases}\mathbf{Z} & (n=0) \\ (\mathbf{Z} / 2 \mathbf{Z})^{2 n+1} & (n \geq 1)\end{cases}
$$

\section{Ring Structure}

We maintain the notations in Sections 1 and 2. In this section, we determine the ring structure of the Hochschild cohomology ring $H H^{*}(\Gamma)$.

Recall the Yoneda product in $H H^{*}(\Gamma)$. Let $\alpha \in H H^{n}(\Gamma)$ and $\beta \in$ $H H^{m}(\Gamma)$, where $\alpha$ and $\beta$ are represented by cocycles $f_{\alpha}: Y_{n} \rightarrow \Gamma$ and $f_{\beta}: Y_{m} \rightarrow$ $\Gamma$, respectively. We have the commutative diagram of $\Gamma^{\mathrm{e}}$-modules with exact rows: 


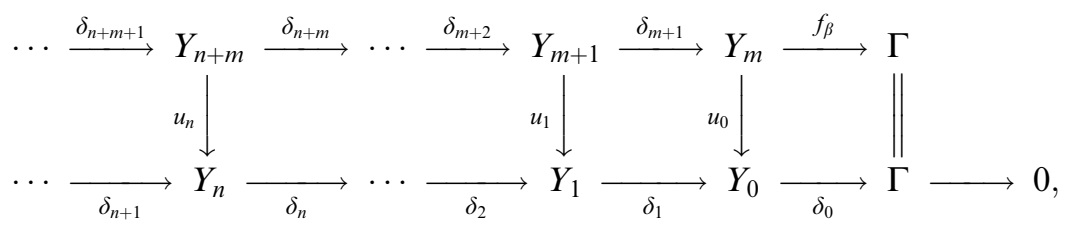

where $u_{l}(0 \leq l \leq n)$ are liftings of $f_{\beta}$. We define the product $\alpha \cdot \beta \in H H^{n+m}(\Gamma)$ by the cohomology class of $f_{\alpha} u_{n}$. This product is independent of the choice of representatives $f_{\alpha}$ and $f_{\beta}$, and liftings $u_{l}(0 \leq l \leq n)$.

By Theorem 2.1, we take generators of $H H^{1}(\Gamma)$ as follows:

$$
\lambda_{1}=i j l_{1}^{1}, \quad \mu_{1}=i j l_{1}^{2}, \quad v_{1}=a^{\prime} j l_{1}^{1}-b^{\prime} i l_{1}^{2} .
$$

Then we have $2 \lambda_{1}=2 \mu_{1}=2 d v_{1}=0$, and $\lambda_{1}, \mu_{1}, v_{1}$ are represented by the following $\Gamma^{\mathrm{e}}$-homomorphisms, respectively:

$$
\begin{aligned}
& \hat{\lambda}_{1}: Y_{1} \rightarrow \Gamma ; \quad c_{1}^{1} \mapsto i j, \quad c_{1}^{2} \mapsto 0, \\
& \hat{\mu}_{1}: Y_{1} \rightarrow \Gamma ; \quad c_{1}^{1} \mapsto 0, \quad c_{1}^{2} \mapsto i j, \\
& \hat{v}_{1}: Y_{1} \rightarrow \Gamma ; \quad c_{1}^{1} \mapsto a^{\prime} j, \quad c_{1}^{2} \mapsto-b^{\prime} i .
\end{aligned}
$$

We state an initial part of liftings of these cocycles.

Lemma 3.1. (i) An initial part of a lifting $u_{n}: Y_{n+1} \rightarrow Y_{n}$ of $\hat{\lambda}_{1}$ is as follows:

$$
\begin{aligned}
& u_{0}\left(c_{1}^{1}\right)=i j c_{0}^{1}, \quad u_{0}\left(c_{1}^{2}\right)=0 ; \\
& u_{1}\left(c_{2}^{1}\right)=-i j c_{1}^{1}, \quad u_{1}\left(c_{2}^{2}\right)=-i j c_{1}^{2}, \quad u_{1}\left(c_{2}^{3}\right)=0 .
\end{aligned}
$$

(ii) An initial part of a lifting $v_{n}: Y_{n+1} \rightarrow Y_{n}$ of $\hat{\mu}_{1}$ is as follows:

$$
\begin{aligned}
& v_{0}\left(c_{1}^{1}\right)=0, \quad v_{0}\left(c_{1}^{2}\right)=i j c_{0}^{1} \\
& v_{1}\left(c_{2}^{1}\right)=0, \quad v_{1}\left(c_{2}^{2}\right)=-i j c_{1}^{1}, \quad v_{1}\left(c_{2}^{3}\right)=-i j c_{1}^{2} .
\end{aligned}
$$

(iii) An initial part of a lifting $w_{n}: Y_{n+1} \rightarrow Y_{n}$ of $\hat{v}_{1}$ is as follows:

$$
\begin{aligned}
& w_{0}\left(c_{1}^{1}\right)=a^{\prime} j c_{0}^{1}, \quad w_{0}\left(c_{1}^{2}\right)=-b^{\prime} i c_{0}^{1} \\
& w_{1}\left(c_{2}^{1}\right)=-a^{\prime} j c_{1}^{1}, \quad w_{1}\left(c_{2}^{2}\right)=b^{\prime} i c_{1}^{1}-a^{\prime} j c_{1}^{2}, \quad w_{1}\left(c_{2}^{3}\right)=b^{\prime} i c_{1}^{2} .
\end{aligned}
$$


Proof. (i) Clearly $\hat{\lambda}_{1}=\delta_{0} u_{0}$ holds. Since

$$
\begin{aligned}
& u_{0} \delta_{2}\left(c_{2}^{1}\right)=a j c_{0}^{1}+i j c_{0}^{1} i=-i j\left(i c_{0}^{1}-c_{0}^{1} i\right)=\delta_{1} u_{1}\left(c_{2}^{1}\right), \\
& u_{0} \delta_{2}\left(c_{2}^{2}\right)=-b i c_{0}^{1}+i j c_{0}^{1} j=-i j\left(j c_{0}^{1}-c_{0}^{1} j\right)=\delta_{1} u_{1}\left(c_{2}^{2}\right), \\
& u_{0} \delta_{2}\left(c_{2}^{3}\right)=0=\delta_{1} u_{1}\left(c_{2}^{3}\right),
\end{aligned}
$$

we have $u_{0} \delta_{2}=\delta_{1} u_{1}$. (ii) and (iii) are similar.

Proposition 3.2. The following equations hold in $H H^{2}(\Gamma)$ :

$$
\begin{gathered}
\lambda_{1}^{2}=a b l_{2}^{1}, \quad \lambda_{1} \mu_{1}=a b l_{2}^{2}, \quad \mu_{1}^{2}=a b l_{2}^{3}, \\
\lambda_{1} v_{1}=a^{\prime} b\left(i l_{2}^{1}+j l_{2}^{2}\right), \quad \mu_{1} v_{1}=a^{\prime} b\left(i l_{2}^{2}+j l_{2}^{3}\right), \quad a^{\prime} \lambda_{1}^{2}+b^{\prime} \mu_{1}^{2}+d v_{1}^{2}=0 .
\end{gathered}
$$

In particular, in the case $a= \pm 1$ and $b= \pm 1, H H^{2}(\Gamma)$ is generated by the products of $\lambda_{1}, \mu_{1}$, and $v_{1}$.

Proof. We calculate $\lambda_{1} v_{1}$ as an example. Since

$$
\begin{aligned}
& \hat{\lambda}_{1} w_{1}\left(c_{2}^{1}\right)=\hat{\lambda}_{1}\left(-a^{\prime} j c_{1}^{1}\right)=a^{\prime} b i, \\
& \hat{\lambda}_{1} w_{1}\left(c_{2}^{2}\right)=\hat{\lambda}_{1}\left(b^{\prime} i c_{1}^{1}-a^{\prime} j c_{1}^{2}\right)=a b^{\prime} j=a^{\prime} b j, \\
& \hat{\lambda}_{1} w_{1}\left(c_{2}^{3}\right)=\hat{\lambda}_{1}\left(b^{\prime} i c_{1}^{2}\right)=0,
\end{aligned}
$$

it follows that $\lambda_{1} v_{1}=a^{\prime} b\left(i l_{2}^{1}+j l_{2}^{2}\right)$ holds. Other computations are similar.

In the following we take generators of $H H^{2}(\Gamma)$ as follows:

$$
\begin{gathered}
\tau_{2}=l_{2}^{1}, \quad v_{2}=l_{2}^{2}, \quad \xi_{2}=l_{2}^{3}, \\
\lambda_{2}=i l_{2}^{1}+j l_{2}^{2}, \quad \mu_{2}=i l_{2}^{2}+j l_{2}^{3} .
\end{gathered}
$$

Then $\tau_{2}, v_{2}, \xi_{2}, \lambda_{2}$, and $\mu_{2}$ are represented by the following $\Gamma^{\mathrm{e}}$-homomorphisms, respectively:

$$
\begin{aligned}
& \hat{\tau}_{2}: Y_{2} \rightarrow \Gamma ; \quad c_{2}^{1} \mapsto 1, \quad c_{2}^{2} \mapsto 0, \quad c_{2}^{3} \mapsto 0 ; \\
& \hat{v}_{2}: Y_{2} \rightarrow \Gamma ; \quad c_{2}^{1} \mapsto 0, \quad c_{2}^{2} \mapsto 1, \quad c_{2}^{3} \mapsto 0 ; \\
& \hat{\xi}_{2}: Y_{2} \rightarrow \Gamma ; \quad c_{2}^{1} \mapsto 0, \quad c_{2}^{2} \mapsto 0, \quad c_{2}^{3} \mapsto 1 ; \\
& \hat{\lambda}_{2}: Y_{2} \rightarrow \Gamma ; \quad c_{2}^{1} \mapsto i, \quad c_{2}^{2} \mapsto j, \quad c_{2}^{3} \mapsto 0 ; \\
& \hat{\mu}_{2}: Y_{2} \rightarrow \Gamma ; \quad c_{2}^{1} \mapsto 0, \quad c_{2}^{2} \mapsto i, \quad c_{2}^{3} \mapsto j .
\end{aligned}
$$


Remark 3.3. By Theorem 2.1 and Proposition 3.2, we have the following:

$$
\begin{gathered}
2 a \tau_{2}=2 b \xi_{2}=2 \lambda_{2}=2 \mu_{2}=2 d v_{2}=0 \\
\lambda_{1}^{2}=a b \tau_{2}, \quad \lambda_{1} \mu_{1}=a b v_{2}, \quad \mu_{1}^{2}=a b \xi_{2}, \quad \lambda_{1} v_{1}=a^{\prime} b \lambda_{2}, \quad \mu_{1} v_{1}=a^{\prime} b \mu_{2} .
\end{gathered}
$$

Note that, since $H H^{*}(\Gamma)$ is graded-commutative and $2 a b v_{2}=0$, it follows that $\mu_{1} \lambda_{1}=-\lambda_{1} \mu_{1}=-a b v_{2}=a b v_{2}=\lambda_{1} \mu_{1}$ hold. Similarly we have $v_{1} \lambda_{1}=\lambda_{1} v_{1}$ and $v_{1} \mu_{1}=\mu_{1} v_{1}$.

Next we state liftings of $\tau_{2}, \xi_{2}, v_{2}, \lambda_{2}$, and $\mu_{2}$.

Lemma 3.4. (i) A lifting $f_{n}: Y_{n+2} \rightarrow Y_{n}$ of $\hat{\tau}_{2}$ is given by $f_{n}\left(c_{n+2}^{k}\right)=c_{n}^{k}$ for $n \geq 0$.

(ii) A lifting $g_{n}: Y_{n+2} \rightarrow Y_{n}$ of $\hat{\xi}_{2}$ is given by $g_{n}\left(c_{n+2}^{k}\right)=c_{n}^{k-2}$ for $n \geq 0$.

(iii) A lifting $h_{n}: Y_{n+2} \rightarrow Y_{n}$ of $\hat{v}_{2}$ is given by $h_{n}\left(c_{n+2}^{k}\right)=c_{n}^{k-1}$ for $n \geq 0$.

(iv) A lifting $r_{n}: Y_{n+2} \rightarrow Y_{n}$ of $\hat{\lambda}_{2}$ is given by $r_{n}\left(c_{n+2}^{k}\right)=j c_{n}^{k-1}+i c_{n}^{k}$ for $n \geq 0$.

(v) A lifting $t_{n}: Y_{n+2} \rightarrow Y_{n}$ of $\hat{\mu}_{2}$ is given by $t_{n}\left(c_{n+2}^{k}\right)=j c_{n}^{k-2}+i c_{n}^{k-1}$ for $n \geq 0$.

Proof. (i) Clearly $\hat{\tau}_{2}=\delta_{0} f_{0}$ holds. If $n(\geq 1)$ is odd, then

$$
\begin{aligned}
f_{n} \delta_{n+3}\left(c_{n+3}^{k}\right) & =f_{n}\left(i c_{n+2}^{k}+c_{n+2}^{k} i+j c_{n+2}^{k-1}+c_{n+2}^{k-1} j\right) \\
& =i c_{n}^{k}+c_{n}^{k} i+j c_{n}^{k-1}+c_{n}^{k-1} j=\delta_{n} f_{n+1}\left(c_{n+3}^{k}\right) .
\end{aligned}
$$

If $n(\geq 2)$ is even, then

$$
\begin{aligned}
f_{n} \delta_{n+3}\left(c_{n+3}^{k}\right) & =f_{n}\left(i c_{n+2}^{k}-c_{n+2}^{k} i+j c_{n+2}^{k-1}-c_{n+2}^{k-1} j\right) \\
& =i c_{n}^{k}-c_{n}^{k} i+j c_{n}^{k-1}-c_{n}^{k-1} j=\delta_{n} f_{n+1}\left(c_{n+3}^{k}\right) .
\end{aligned}
$$

Thus (i) is proved. Other computations are similar.

REMARK 3.5. Let $\alpha=\sum_{k=1}^{n+1} \gamma_{k} l_{n}^{k}\left(\gamma_{k} \in \Gamma\right)$ be any element in $H H^{n}(\Lambda)$ for $n \geq 1$. Then by Lemma 3.4 we have

$$
\alpha \tau_{2}=\sum_{k=1}^{n+1} \gamma_{k} l_{n+2}^{k}, \quad \alpha \xi_{2}=\sum_{k=1}^{n+1} \gamma_{k} l_{n+2}^{k+2}, \quad \alpha v_{2}=\sum_{k=1}^{n+1} \gamma_{k} l_{n+2}^{k+1} .
$$

By using this remark, it is shown that $H H^{3}(\Gamma)$ is generated by products of $\lambda_{1}, \mu_{1}, v_{1}, \tau_{2}, v_{2}$, and $\xi_{2}$ : 


$$
\begin{gathered}
\lambda_{1} \tau_{2}=i j l_{3}^{1}, \quad \mu_{1} \tau_{2}\left(=\lambda_{1} v_{2}\right)=i j l_{3}^{2}, \quad \lambda_{1} \xi_{2}\left(=\mu_{1} v_{2}\right)=i j l_{3}^{3}, \quad \mu_{1} \xi_{2}=i j l_{3}^{4}, \\
v_{1} \tau_{2}=a^{\prime} j l_{3}^{1}-b^{\prime} i l_{3}^{2}, \quad v_{1} v_{2}=a^{\prime} j l_{3}^{2}-b^{\prime} i l_{3}^{3}, \quad v_{1} \xi_{2}=a^{\prime} j l_{3}^{3}-b^{\prime} i l_{3}^{4} .
\end{gathered}
$$

We state the relations in degree 3, which are given by using Lemma 3.4.

Proposition 3.6. The following relations hold in $H H^{3}(\Gamma)$ :

$$
\begin{gathered}
\mu_{1} \tau_{2}=\lambda_{1} v_{2}, \quad \lambda_{1} \xi_{2}=\mu_{1} v_{2}, \quad \lambda_{1} \mu_{2}=\mu_{1} \lambda_{2}=d v_{1} v_{2}, \quad \lambda_{1} \lambda_{2}=d v_{1} \tau_{2}, \\
\mu_{1} \mu_{2}=d v_{1} \xi_{2}, \quad v_{1} \lambda_{2}=a^{\prime} \lambda_{1} \tau_{2}+b^{\prime} \lambda_{1} \xi_{2}, \quad v_{1} \mu_{2}=a^{\prime} \mu_{1} \tau_{2}+b^{\prime} \mu_{1} \xi_{2} .
\end{gathered}
$$

Likewise, we may show that $H H^{4}(\Gamma)$ is generated by products of $\tau_{2}, v_{2}, \xi_{2}$, $\lambda_{2}, \mu_{2}$ :

$$
\begin{gathered}
\tau_{2}^{2}=l_{4}^{1}, \quad \tau_{2} v_{2}=l_{4}^{2}, \quad \tau_{2} \xi_{2}\left(=v_{2}^{2}\right)=l_{4}^{3}, \quad v_{2} \xi_{2}=l_{4}^{4}, \quad \xi_{2}^{2}=l_{4}^{5}, \\
\lambda_{2} \tau_{2}=i i_{4}^{1}+j l_{4}^{2}, \quad \mu_{2} \tau_{2}\left(=\lambda_{2} v_{2}\right)=i l_{4}^{2}+j l_{4}^{3}, \\
\lambda_{2} \xi_{2}\left(=\mu_{2} v_{2}\right)=i l_{4}^{3}+j l_{4}^{4}, \quad \mu_{2} \xi_{2}=i i_{4}^{4}+j l_{4}^{5} .
\end{gathered}
$$

We state the relations in degree 4 , which are given by using Lemma 3.4.

Proposition 3.7. The following relations hold in $\mathrm{HH}^{4}(\Gamma)$ :

$$
\begin{gathered}
\tau_{2} \xi_{2}=v_{2}^{2}, \quad \mu_{2} \tau_{2}=\lambda_{2} v_{2}, \quad \lambda_{2} \xi_{2}=\mu_{2} v_{2}, \\
\lambda_{2}^{2}=a \tau_{2}^{2}+b \tau_{2} \xi_{2}, \quad \lambda_{2} \mu_{2}=a \tau_{2} v_{2}+b v_{2} \xi_{2}, \quad \mu_{2}^{2}=a \tau_{2} \xi_{2}+b \xi_{2}^{2} .
\end{gathered}
$$

Similarly, by using Remark 3.5, it is not hard to see that $H H^{n}(\Gamma)$ for $n \geq 5$ is multiplicatively generated by products of $\lambda_{1}, \mu_{1}, v_{1}, \tau_{2}, v_{2}, \xi_{2}, \lambda_{2}$, and $\mu_{2}$.

Now suppose that $\mathscr{A}=\mathbf{Z}\left[X_{1,1}, X_{1,2}, X_{1,3}, X_{2,1}, X_{2,2}, X_{2,3}, X_{2,4}, X_{2,5}\right] \quad$ is a graded algebra with $\operatorname{deg} X_{k, \ell}=k$ for $k=1,2$. We consider the algebra homomorphism $\Phi: \mathscr{A} \rightarrow H H^{*}(\Gamma)$ induced by $X_{k, 1} \mapsto \lambda_{k}, \quad X_{k, 2} \mapsto \mu_{k}, \quad X_{k, 3} \mapsto v_{k}$, $X_{2,4} \mapsto \tau_{2}$, and $X_{2,5} \mapsto \xi_{2}$ where $k=1,2$. Let $\mathscr{I}$ denote the set of the relations $2 \lambda_{1}=2 \mu_{1}=2 d v_{1}=0$ and the relations given by Propositions 3.2, 3.6, and 3.7 and Remark 3.3. We rewrite $\mathscr{I}$ by the correspondence $\theta$ which is defined by $\lambda_{k} \mapsto X_{k, 1}, \mu_{k} \mapsto X_{k, 2}, v_{k} \mapsto X_{k, 3}, \tau_{2} \mapsto X_{2,4}$, and $\xi_{2} \mapsto X_{2,5}$ where $k=1,2$, and denote it by $\theta(\mathscr{I})$. The algebra homomorphism $\Phi$ induces a surjective algebra homomorphism $\tilde{\Phi}: \mathscr{B}=\mathscr{A} / \theta(\mathscr{I}) \rightarrow H H^{*}(\Gamma)$. Let $\mathscr{B}_{n}=\{z \in \mathscr{B} \mid \operatorname{deg} z=n\}$ for $n>0$.

If $n=1, \mathscr{B}_{1}$ is additively generated by $X_{1,1}, X_{1,2}, X_{1,3}$. Then $X_{1,1}$ and $X_{1,2}$ have order dividing 2 , and $X_{1,3}$ has order dividing $2 d$. Thus the order of $\mathscr{B}_{1}$ is at most $2^{2} \cdot 2 d$. 
If $n=2, \mathscr{B}_{2}$ is additively generated by $X_{2,1}, X_{2,2}, X_{2,3}, X_{2,4}$, and $X_{2,5}$. Then $X_{2,1}, X_{2,2}, X_{2,3}, X_{2,4}, X_{2,5}$ have order dividing $2,2,2 d, 2 a, 2 b$, respectively. Thus the order of $\mathscr{B}_{2}$ is at most $2^{2} \cdot 2 d \cdot 2 a \cdot 2 b$.

If $n=2 k+1(k \neq 0)$, then $\mathscr{B}_{n}$ is additively generated by

$$
X_{1, \ell} X_{2,4}^{k-s} X_{2,5}^{s} \quad(\ell=1,2,3,0 \leq s \leq k), \quad X_{1,3} X_{2,3} X_{2,4}^{k-s} X_{2,5}^{s-1} \quad(1 \leq s \leq k) .
$$

Thus $X_{1, \ell} X_{2,4}^{k-s} X_{2,5}^{s}(\ell=1,2,0 \leq s \leq k)$ have order dividing 2 , and the other generators have order dividing $2 d$. Thus the order of $\mathscr{B}_{n}$ is at most $2^{n+1} \cdot(2 d)^{n}$.

If $n=2 k(k \geq 2)$, then $\mathscr{B}_{n}$ is additively generated by

$$
X_{2,4}^{k-s} X_{2,5}^{s} \quad(0 \leq s \leq k), \quad X_{2, \ell} X_{2,4}^{k-s} X_{2,5}^{s-1} \quad(\ell=1,2,3,1 \leq s \leq k) .
$$

Then $X_{2,4}^{k}$ has order dividing $2 a, X_{2,5}^{k}$ has order dividing $2 b, X_{2, \ell} X_{2,4}^{k-s} X_{2,5}^{s-1}$ $(\ell=1,2,1 \leq s \leq k)$ have order dividing 2 , and the other generators have order dividing $2 d$. Note that for $1 \leq s \leq k-1, X_{2,4}^{k-s} X_{2,5}^{s}$ has order dividing $2 d$, because $X_{2,3}^{2}=X_{2,4} X_{2,5}$ and $X_{2,3}$ has order dividing $2 d$. Thus the order of $\mathscr{B}_{n}$ is at most $2^{n} \cdot 2 a \cdot 2 b \cdot(2 d)^{n-1}$.

Hence the order of $\mathscr{B}_{n}$ is at most the order of $H H^{n}(\Gamma)$ for $n>0$ (see Theorem 2.1). Therefore $\tilde{\Phi}: \mathscr{B} \rightarrow H H^{*}(\Gamma)$ is also injective.

Finally, we state the ring structure of $H H^{*}(\Gamma)$.

THEOREM 3.8. The Hochschild cohomology ring $H H^{*}(\Gamma)$ is the commutative graded ring which is generated by the elements

$$
\lambda_{1}, \mu_{1}, v_{1} \in H H^{1}(\Gamma), \quad \tau_{2}, \xi_{2}, v_{2}, \lambda_{2}, \mu_{2} \in H H^{2}(\Gamma),
$$

and is defined by the following relations:

(i) degree-1 relations

$$
2 \lambda_{1}=2 \mu_{1}=2 d v_{1}=0 .
$$

(ii) degree-2 relations

$$
\begin{gathered}
2 a \tau_{2}=2 b \xi_{2}=2 \lambda_{2}=2 \mu_{2}=2 d v_{2}=0, \quad a^{\prime} \lambda_{1}^{2}+b^{\prime} \mu_{1}^{2}+d v_{1}^{2}=0, \\
\lambda_{1}^{2}=a b \tau_{2}, \quad \lambda_{1} \mu_{1}=a b v_{2}, \quad \mu_{1}^{2}=a b \xi_{2}, \quad \lambda_{1} v_{1}=a^{\prime} b \lambda_{2}, \quad \mu_{1} v_{1}=a^{\prime} b \mu_{2} .
\end{gathered}
$$

(iii) degree-3 relations

$$
\begin{aligned}
& \mu_{1} \tau_{2}=\lambda_{1} v_{2}, \quad \lambda_{1} \xi_{2}=\mu_{1} v_{2}, \quad \lambda_{1} \mu_{2}=\mu_{1} \lambda_{2}=d v_{1} v_{2}, \quad \lambda_{1} \lambda_{2}=d v_{1} \tau_{2} \\
& \mu_{1} \mu_{2}=d v_{1} \xi_{2}, \quad v_{1} \lambda_{2}=a^{\prime} \lambda_{1} \tau_{2}+b^{\prime} \lambda_{1} \xi_{2}, \quad v_{1} \mu_{2}=a^{\prime} \mu_{1} \tau_{2}+b^{\prime} \mu_{1} \xi_{2}
\end{aligned}
$$


(iv) degree-4 relations

$$
\begin{gathered}
\tau_{2} \xi_{2}=v_{2}^{2}, \quad \mu_{2} \tau_{2}=\lambda_{2} v_{2}, \quad \lambda_{2} \xi_{2}=\mu_{2} v_{2}, \\
\lambda_{2}^{2}=a \tau_{2}^{2}+b \tau_{2} \xi_{2}, \quad \lambda_{2} \mu_{2}=a \tau_{2} v_{2}+b v_{2} \xi_{2}, \quad \mu_{2}^{2}=a \tau_{2} \xi_{2}+b \xi_{2}^{2} .
\end{gathered}
$$

Remark 3.9. The result of Sanada [6, Section 3.4] follows from Theorem 3.8:

If $a= \pm 1$ and $b= \pm 1$, then $\tau_{2}, \xi_{2}, v_{2}, \lambda_{2}, \mu_{2}$ are generated by the products of $\lambda_{1}, \mu_{1}$, and $v_{1}$. Hence $H H^{*}(\Gamma)$ is the commutative graded ring which is generated by $\lambda_{1}, \mu_{1}, v_{1} \in H H^{1}(\Gamma)$, and is defined by the following relations:

$$
2 \lambda_{1}=2 \mu_{1}=2 v_{1}=0, \quad \lambda_{1}^{2}+\mu_{1}^{2}+v_{1}^{2}=0 .
$$

\section{Acknowledgements}

The author would like to express his gratitude to the referee for valuable comments and suggestions.

\section{References}

[1] Cartan, H. and Eilenberg, S., Homological Algebra, Princeton University Press, Princeton NJ, 1956.

[2] Hayami, T., Hochschild cohomology ring of an order of a simple component of the rational group ring of the generalized quaternion group, Comm. Algebra 36 (2008), 2785-2803.

[ 3 ] Hayami, T., Hochschild cohomology ring of a maximal order of the quaternion algebra, Tsukuba J. Math. 36 (2012), 111-120.

[4] Hochschild, G., On the Cohomology Groups of an Associative Algebra, Ann. of Math. 46 (1945), 58-67.

[ 5 ] MacLane, S., Homology, Springer-Verlag, New York, 1975.

[6] Sanada, K., On the Hochschild cohomology of crossed products, Comm. Algebra 21 (1993), 2727-2748.

Department of Mathematics

Hokkai-Gakuen University

4-1-40, Asahi-machi, Toyohira-ku, Sapporo 062-8605

Japan

E-mail: hayami@ma.kagu.tus.ac.jp 